\title{
A readership survey of Western Pacific Surveillance and Response Journal
}

\author{
Michelle McPherson, ${ }^{a}$ Elizabeth Mangali, a James Fielding, ${ }^{b}$ Joy Gregory a and Ailan Lic \\ Correspondence to the Coordinating Editor (email: WPSAR@wpro.who.int).
}

W e established the Western Pacific Surveillance and Response Journal (WPSAR) in 2010 to increase the dissemination of data from surveillance systems in the Asia Pacific region as part of the Asia Pacific Strategy for Emerging Diseases. ${ }^{1}$ WPSAR was to provide a platform for people working in surveillance and response in the Western Pacific Region to share scientific and operational findings and publish a broad range of articles not limited to conventional research articles.

In mid-2014, four years after the first issue of WPSAR, an online survey of WPSAR subscribers was conducted to assess the impact, network and visibility of WPSAR in the region to determine if these objectives had been met. Based on a similar survey undertaken by Eurosurveillance in $2011,^{2}$ we sought to understand the WPSAR audience more comprehensively, how the journal is used and readers' expectations. The WPSAR readership survey link was emailed to the 514 registered subscribers, and $25 \%$ responded.

The readership survey indicated that the profile, visibility and readership of WPSAR is growing; nearly half of the responders reported reading their first WPSAR article in the previous 12 months. The journal also has considerable reach to 28 countries around the world, with more readers from Australia and the Philippines. Respondents worked in 16 countries from the World Health Organization's Western Pacific Region with others in Africa, Europe, South-East Asia and North America. Most of our readers work in public health practice and/or field epidemiology as epidemiologists, disease surveillance officers and public health specialists primarily for government, academic institutions or for public health organizations - our targeted audience.
Responses generally indicated satisfaction with the content, delivery, operation and expectations of WPSAR, and the journal is regarded as useful. Originality of content and timeliness were not rated as high; the latter is surprising as we take an average of only three months to publish our articles from their submission. We were pleased that our articles were rated as easy to read with clear figures, tables and illustrations; that there is an ease of access; and that expectations of the journal rated high.

That the journal is indexed in PubMed and has a regional scope were important for attracting manuscripts and readership, but further improvement by having an impact factor was a common theme. We are currently being evaluated for an impact factor and look forward to the result. That two thirds of respondents were aware that WPSAR is indexed on PubMed is encouraging, as is the 2000 times a month WPSAR articles are accessed through PubMed. ${ }^{3}$

A wide range of article types is included in WPSAR such as outbreak investigations, surveillance reports and evaluations and lessons from the field. This is in addition to the more standard original research articles. Although there has been an even distribution of these different article types in WPSAR, more than half of survey responders reported they read outbreak investigation and surveillance reports the most, suggesting that our second objective is being met.

At the time of the survey, 14 issues of WPSAR had been published comprising 101 non-editorial articles with 43 different subjects addressed. The most common of these were influenza, dengue, emergency response and tuberculosis; these topics are most likely linked

\footnotetext{
WPSAR Editorial Team, Division of Health Security and Emergencies, World Health Organization Regional Office for the Western Pacific, Manila, Philippines.

Emerging Disease Surveillance and Response Unit, Division of Health Security and Emergencies, World Health Organization Regional Office for the Western Pacific, Manila, Philippines.

WPSAR Executive Editor, Division of Health Security and Emergencies, World Health Organization Regional Office for the Western Pacific, Manila, Philippines. 
to our original policy of assigning themes to issues prospectively. All four topics were an assigned theme - for example, the emergency response articles refer to a themed issue on the Great Japan Earthquake. Since 2014, the number of non-themed submissions has increased, and we now publish a broader range of topics.

It was also noted that most of the WPSAR articles were on infectious diseases (with the exception of the 'Great East Japan Earthquake' issue), even though the scope of WPSAR is all activities related to the surveillance of and response to public health events and emergencies. As WPSAR represents a Region with an alarming number of disasters, including regular typhoons in the Philippines and the recent earthquake in Vanuatu, we encourage submissions on responding to these events. In fact we will be publishing a special issue on responding to Typhoon Haiyan later this year.

There were two suggestions from the survey that corroborate our long-term strategy for WPSAR. That very few respondents - even among the relatively few Chinese speakers who participated in the survey - knew that WPSAR is published in Mandarin suggests that we can greatly increase our visibility in China. Also, several respondents suggested broadening the scope of WPSAR to include noncommunicable diseases which is part of our existing long-term future.

Although we are pleased with the results of the survey that suggest that WPSAR is meeting its objectives, we recognize that the sample size may not be representative of all our subscribers or all those who read our articles either on PubMed or from our own website. However, our response rate of $25 \%$ does favourably compare to that of others journals which ranged from $7 \%$ to $43 \%$. $^{3-8}$
Therefore, in the relatively short time since its inception, WPSAR has established itself as a good quality regional journal that is well regarded by its readership. That two thirds of readers think it is fulfilling its role for timely sharing of information and that there is a wide range of article types and topics being published suggest that we are meeting our objectives of providing a platform for information sharing in surveillance and response in the Western Pacific Region.

We thank the survey responders and all of our subscribers, authors and reviewers: without you there would be no WPSAR. We look forward to WPSAR continuing to be the platform for publishing your surveillance and response work.

\section{References:}

1. Asia Pacific Strategy for Emerging Diseases. (2005). Manila, World Health Organization Regional Office for the Western Pacific, 2005 (http://www.wpro.who.int/emerging_diseases/documents/ APSED final endorsed and edited by EDT-map removed FORMAT̄-20/en/, accessed 5 Jüne 2015).

2. Steffens I. The Eurosurveillance reader survey - what's next? Euro Surveillance: European Communicable Disease Bulletin, 2011 16: $\mathrm{pii}=20014$ (http://www.eurosurveillance.org/ViewArticle. aspx?Articleld=20014, accessed 15 May 2015).

3. PubMed Central Publisher Services. Maryland, National Institutes of Health, 2015.

4. Stein KF. Readership survey results. Journal of the American Psychiatric Nurses Association, 2008, 14:33-35. doi:10.1177/1078390307313916 pmid:21672878

5. Haines GR, Hillman BJ. The 2012 JACR readership survey. Journal of the American College of Radiology: JACR, 2013, 10:234-236. doi:10.1016/j.jacr.2013.01.010 pmid:23545080

6. Joshua E. Readership survey 2011. Journal of Oral and Maxillofacial Pathology, 2012, 16:1-3. doi:10.4103/0973029X.92964 pmid:22438636

7. Starr S. Journal of the Medical Library Association readership survey. Journal of the Medical Library Association: JMLA, 2013, 101:167. doi:10.3163/1536-5050.101.3.001 pmid:23930083

8. Day PC. Readership survey 2013: what you think about our journal. Aviation, Space, and Environmental Medicine, 2013, 84:639641. doi:10.3357/ASEM.3720.2013 pmid:23745295 